\title{
Prevention of Cervical Cancer with Vaccines
}

\author{
Samuel S. Im, MD, Bradley J. Monk, MD, and Luis P. Villarreal, PhD
}

\begin{abstract}
Address
Department of Molecular Biology and Biochemistry, School of Biological Sciences, University of California, Irvine, Bio Sciences II, Room 3232, Irvine, CA 92697, USA. E-mail: bjmonk@uci.edu

Current Oncology Reports 200I, 3:322-328

Current Science Inc. ISSN I523-3790

Copyright $(200$ I by Current Science Inc.
\end{abstract}

Worldwide, cervical cancer is one of the most common cancers in women. This is especially true in developing countries, where Papanicolaou smear screening, an effective preventive measure against cervical cancer, is insufficiently implemented. With growing evidence for human papillomavirus as a central etiologic factor in cervical neoplasia, development of a vaccine against this virus has emerged as an important objective in prevention of cervical cancer. International efforts in vaccine development have culminated in advancement of various vaccine strategies and initiation of human clinical trials. Reports from animal vaccine trials and early phase I human trials indicate markedly enhanced immune response through vaccination. However, the clinical significance of these results requires confirmation from long-term human trials.

\section{Introduction}

Cervical cancer is the second most common cancer among women [1]. About 450,000 women are newly diagnosed with cervical cancer each year around the world, and another 200,000 women succumb to this disease [2,3]. The development of cervical cancer is strongly associated with human papillomavirus (HPV) infection. HPV is detected in more than $99 \%$ of cervical cancers and is considered one of the main factors in carcinogenesis [4].

HPV belongs to the Papovaviridae family of viruses and has a tropism for epithelia at specific body locations. Over 100 different HPV genotypes have been cloned and sequenced [5]. Among these, about 35 HPV types infect the female genital tract $[6 \bullet]$. HPV types 6 and 11 are usually associated with common genital condyloma and thus pose a low risk for invasive cervical carcinoma. HPV types 16 , $18,31,33,45,52$, and 58 carry a higher risk for cervical cancer. In fact, HPV 16 is detected in about half of all squamous-cell cervical cancers [7], and it is the main focus for a great deal of research in cervical cancer and HPV.
HPV is a double-stranded circular DNA virus with a genome of about 8000 base pairs (Fig. 1). The HPV genome can be grouped into early and late genes. The early genes, E2, E6, and E7, may play important roles in carcinogenesis. In particular, E6 and E7 are known oncogenes. E6 encodes for a 16 to $19-\mathrm{kD}$ protein that is present at low levels in both the nucleus and the cytoplasm [8]. It binds to the tumor suppressor protein $\mathrm{p} 53$ and causes its degradation by the ubiquitin proteolysis pathway [9]. Also, E6 has been linked to the telomerase activation and immortalization of cells. E7 encodes for a 10 to $14-\mathrm{kD}$ protein that has transforming activity and regulatory functions [8]. It binds pRB (retinoblastoma-susceptibility protein) and modulates cell-cycle control.

The functions of E6 and E7 may differ among the HPV types. The low-risk and high-risk HPV types seem to have differing binding capacity of their E6- and E7-encoded proteins to products of tumor suppressor genes [10]. The low-risk types have very low or no affinity for the p53 and pRB. The higher-risk types appear to have increased binding capacity for these tumor suppressor genes and promote immortalization of cells, possibly leading to tumorigenesis.

The late proteins consist mainly of L1 and L2. L1 is the major capsid protein for the papillomavirus, and it is the most antigenic of the HPV-encoded proteins [8]. L2 is the minor capsid protein. The capsid proteins form the icosahedral capsids with 72 capsomers to enclose the HPV genome [11]. The discovery that these capsid proteins could form virus-like particles without their genome contributed to HPV vaccine development [12]. Prior to this discovery, because HPV could not be easily cultured in the laboratory, attenuated forms of the virus could not be developed as a vaccine. This conventional approach to the development of viral vaccines has not been useful in HPV and has hindered vaccine development for cervical cancer.

\section{HPV Vaccine Strategies}

In cervical cancer vaccine development, the induction of immunity to HPV proteins E6, E7, L1, and L2 plays a crucial role in the strategy against HPV infection and subsequent HPV-related precancerous and cancerous lesions (Tables 1 and 2). Both cellular and humoral immunity are important to mount an immune response against this virus. E6 and E7 expression has been identified in cervical dysplasia and in cervical cancer tissue $[13,14]$. The presence of E6 and E7 


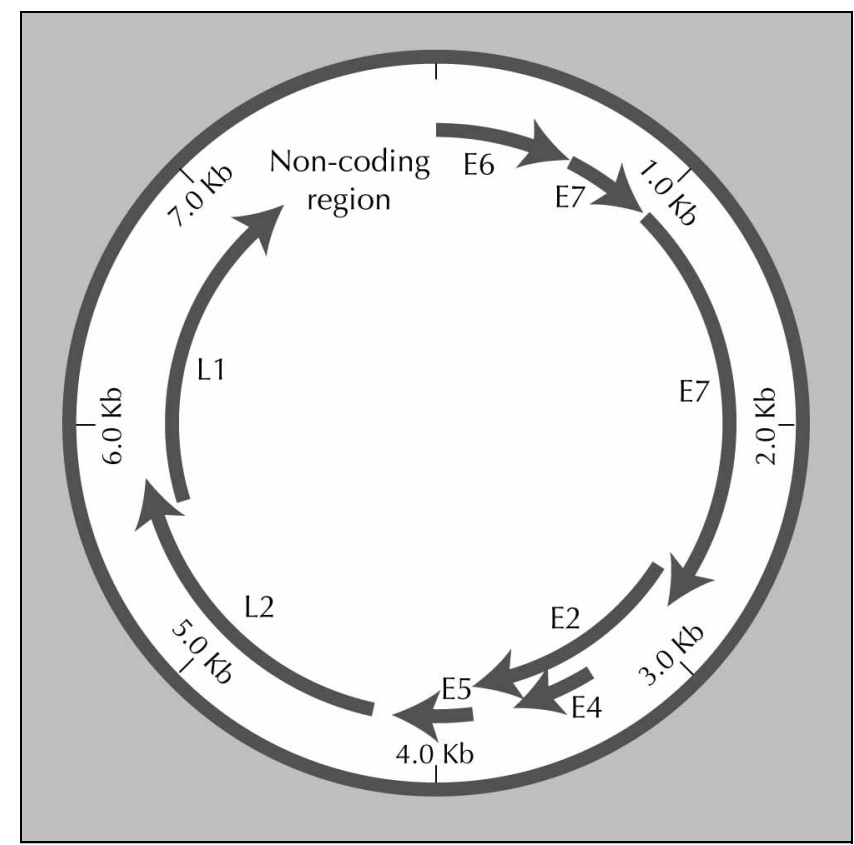

Figure 1. HPV-16 genomic map.

throughout the precancerous and cancerous stages makes them ideal targets for vaccine development. This type of vaccine would be useful in therapy for tissues that are already infected with HPV as well as in primary prevention.

L1 and L2 encode capsid proteins and are known to be highly antigenic [8]. Recent research has focused on using these proteins to elicit a strong humoral immune response from the host. This prophylactic vaccine should elicit antibodies against these capsid antigens and theoretically protect the genital epithelia from HPV infection. This hypothesis is based upon the observation that the natural immune response to HPV infection involves a robust humoral immune response from the host [13]. Recently, af-Geijersstam et al. [15] showed that HPV-16 capsid typespecific antibodies were detected in 33\% of HPV-negative women, 55\% of HPV-infected women with no lesions, and $72 \%$ of adult women with cervical dysplasia. Such antibodies seem to clear HPV from healthy, sexually active women, with only a very small subset of women developing progressive cervical dysplasia. Interestingly, humoral immune suppression does not increase HPV-associated cervical lesions, suggesting that HPV immunity is still incompletely understood [16]. However, in hosts with depressed cellular immune systems, such as HIV and transplant patients, a marked increase in the incidence of HPVrelated precancerous and cancerous lesions is reported [17-19]. Thus, both humoral and cellular immunocompetence seem to be critical to HPV surveillance and clearance.

Vaccine strategies for cervical cancer have been grouped into therapeutic and preventive approaches. The ubiquitous presence of $\mathrm{E} 6$ and $\mathrm{E} 7$ in the cellular cytoplasm during all stages of cervical neoplasia makes these proteins ideal targets for therapeutic vaccines [13]. These vaccines would elicit a cellular immune response and cytotoxic $\mathrm{T}$ lymphocytes (CTL) to eradicate the infected cells and tissue. In contrast, humoral immunity may be more important in preventive vaccines. Vaccines that target HPV capsid proteins and elicit an antigenic reaction from the host through the humoral immune response stimulating mucosal immunoglobulins (IgA and IgG) would be expected to prevent HPV from entering the host.

Ideally, effective HPV vaccination would elicit both humoral and cellular immunity. Indeed, clinical trials and animal model experiments of these vaccines have shown a combination of cellular and humoral immune system induction. Vaccines can also be categorized into peptide and DNA-based strategies. For example, peptide vaccines of early proteins, viral capsid protein vaccines, and E6 and E7 DNA vaccines have been studied. In efforts to augment immunity, various adjuvant agents, such as alum and Freund's adjuvant, have been used to increase the potency and immunogenicity of the molecules.

Animal and Human Trials of HPV Vaccines

Until the development of virus-like particles (VLP), HPV vaccine research was rudimentary and unsuccessful. It was demonstrated that the major capsid protein L1 alone can self-assemble and form an HPV-like viral particle. These particles, which do not contain DNA, not only resembled the wild-type HPV icosahedral virion but also induced high titers of antigenicity when given to animals $[20,21]$. Prior to this discovery, HPV vaccine experiments involved denatured L1 capsid proteins and only elicited minimal immune response within the vaccinated animals $[22,23]$. The main advantage of VLP-based vaccines was that VLPs were readily produced through the use of recombinant bacteria, insect cells, and eukaryotic cells. At the same time, VLPs could elicit high immune response without involving the virulent HPV genome.

Along with the difficulty in culturing HPV in the laboratory, another problem with HPV was that it did not crossinfect other species. This meant that VLPs derived from the human virus strain could not be tested in animals. Subsequent animal studies had to involve animal VLPs with corresponding animal papillomaviruses, such as the cottontail rabbit papillomavirus (CRPV), canine oral papillomavirus (COPV), and bovine papillomavirus type 4 (BPV4). Immune response experiments using animal VLPs showed that VLP vaccination protected the animals from their respective papillomaviruses [24-27]. The protection was durable and lasted at least 1 year.

There was also concern that these parenterally administered vaccines might not produce enough IgG and IgA at the cervicovaginal epithelia to neutralize HPV before infection. Several elegant studies have shown the mucosal effects of VLP vaccines. Nardelli-Haefliger et al. [28] demonstrated that mucosal immunity in both the nasal and genital system could be induced when VLPs were administered 


\begin{tabular}{|c|c|}
\hline Protein & Function \\
\hline EI & $\begin{array}{l}\text { Origin recognition protein; forms a complex } \\
\text { with E2 to facilitate viral replication }\end{array}$ \\
\hline E2 & $\begin{array}{l}\text { Regulates viral DNA transcription and } \\
\text { replication; suppresses the promoter from } \\
\text { which E6 and E7 proteins are transcribed }\end{array}$ \\
\hline E4 & $\begin{array}{l}\text { Contained completely within the E2 open } \\
\text { reading frame; reported to disrupt the } \\
\text { cytokeratin matrix }\end{array}$ \\
\hline E5 & $\begin{array}{l}\text { Small hydrophobic membrane protein; } \\
\text { appears to bind and activate growth factor } \\
\text { receptor }\end{array}$ \\
\hline E6 & $\begin{array}{l}\text { Oncogene; has transforming and } \\
\text { immortalizing functions; suppresses p } 53\end{array}$ \\
\hline E7 & $\begin{array}{l}\text { Oncogene; has transforming and } \\
\text { transcriptional regulatory functions; } \\
\text { suppresses pRB }\end{array}$ \\
\hline $\mathrm{LI}$ & Major capsid protein; highly antigenic \\
\hline L2 & Minor capsid protein \\
\hline
\end{tabular}

intranasally. This concept supports the theory that all body mucosal surfaces share a common immune system, preventing the entrance of pathogens into the body.

Further work on the nasal vaccination with HPV-16 VLPs continued with Dupuy et al. [6•]. These investigators added cholera toxin as an adjuvant and were able to show that, in addition to the IgA and IgG in the murine mucosal secretions, CD4+ and CD8+ activation were also demonstrated, indicating stimulation of both humoral and cellular immune response. Furthermore, Revaz et al. [29] showed that, in addition to prophylactic immunity, the VLP nasal vaccine can also induce antitumor immunity in a murine model. This confirmed the previous finding of dual activation of the cellular and humoral response from the nasal VLP vaccine $[6 \bullet, 30]$. The mechanism of CTL induction appears to be related to VLP processing by antigen-presenting cells. L1 and L2 protein are presented through the major histocompatibility I (MHC) pathway and elicit CD8+ and CTL activation, as noted by Rudolf et al. [30].

An international human VLP clinical trial was recently concluded at The Johns Hopkins University Center for Immunization Research [31••]. This was the first double-blinded, randomized, placebo-controlled, phase I safety and immunogenicity trial of a VLP prophylactic vaccine in humans. Fifty-eight women and 14 men were selected for this trial, with these subjects determined to be at low risk for HPV-16 exposure. Their HPV16 serostatus was determined prior to the start of the vaccination, and six subjects were found to be seropositive. The serologic levels of these six subjects established the baseline natural serologic level for HPV-16 exposure. After vaccination, no adverse complications were noted, and the vaccine was well tolerated. Whether vaccinated with or without the adjuvant, the majority of the vaccinated human subjects showed increased serum antibody titer that was about 40-fold higher than the level in those who were naturally seroconverted. In summary, the vaccine did induce an immune response from the host. The clinical significance of this finding will remain unknown until more trials are completed.

Earlier human vaccine trials have been limited for the most part to patients with advanced cervical cancer and have focused on eliciting a therapeutic response. E6 and E7 were the main targets for these vaccine trials. Borysiewics et al. [32] reported administering live recombinant vaccinia virus expressing the E6 and E7 proteins of HPV-16 and HPV-18 to patients with stage IB to IIIB and recurrent cervical cancer. A total of eight patients were recruited, and none of the participants had any side effects from the vaccination. However, only one patient showed any conclusive specific CTL response for the vaccine. Interestingly, all eight patients mounted an antivaccinia virus antibody reaction, making further immunization with this vector problematic because host immunity would be expected to destroy the vaccine/vector before HPV immunity could develop.

Viral vectors represent one of the major ways to deliver E6 and E7 genes to targeted cells and to promote production of antigenic proteins inside the host. Gene therapy trials have studied many viral vectors, especially adenovirus. These viral vectors became useful in vaccine development. The underlying concept has been that the delivery of genes and translation of viral proteins would induce CTL after MHC I-dependent antigen presentation. Although recombinant genes are used with minimal transformation activity, there is always the risk of DNA integration into the host genome by the oncogenes.

In addition to VLPs and viral vectors, peptide vaccines using E6 and E7 proteins have also been employed in clinical trials. Steller et al. [33] tested the HLA-A*0201restricted, HPV-16 E7 peptide vaccine in a nonrandomized phase I trial involving women with refractory vaginal and cervical cancers. Twelve patients were recruited and were given four inoculations of the vaccine at 3-week intervals, with no apparent side effects. Although no clinical response from the vaccination was reported, this study showed that, even in these advanced-stage patients, a specific cellular immune response could be generated and even be augmented by peptide vaccination. Similarly, van Driel et al. [34] recruited HLA-A*0201-positive patients with HPV-positive cervical carcinoma for a phase I/II clinical trial. HPV-16 E7 peptides with two CTL epitopes were used to vaccinate 19 patients, again with no adverse effects. These findings further bolster the notion that vaccines can be safely carried out and are feasible even in advancedstage cancer patients. However, in a follow-up report by the same group, no specific CTL response against the HPV-16 E7 peptides was reported [35]. 


\begin{tabular}{|c|c|c|}
\hline Vaccine type & Components & Function \\
\hline VLP & LI and L2 capsid proteins & $\begin{array}{l}\text { Mostly induces strong humoral immune } \\
\text { response; main prophylactic vaccine approach }\end{array}$ \\
\hline Peptide & Peptides of CTL epitopes, especially E6 and E7 & $\begin{array}{l}\text { Mostly induces cellular immune response; use of } \\
\text { immunogenic epitopes from E6 and E7 for } \\
\text { therapeutic vaccine }\end{array}$ \\
\hline DNA & $\begin{array}{l}\text { DNA segments that encode for the HPV genes, } \\
\text { especially E6 and E7 }\end{array}$ & $\begin{array}{l}\text { Appears to induce humoral and cellular immunity } \\
\text { when different vaccine potency enhancement } \\
\text { modalities are used }\end{array}$ \\
\hline
\end{tabular}

Unlike the vaccine trials involving advance-staged cancer patients, Muderspach et al. [36] presented their findings from a peptide vaccine trial in patients with high-grade cervical and vulvar intraepithelial neoplasia. This trial involved patients with precancerous lesions who were HLA-A2-positive and HPV-16-positive. The HPV-16 E7 peptide with incomplete Freund's adjuvant was administered to 18 patients. Results indicate that peptide vaccination in a precancerous lesion can augment cellular immune response. However, the data do not show whether the vaccine can eradicate HPV from the lower genital tract or induce lesion regression.

The MHC restriction requirement of the peptide vaccine makes it an unattractive option when compared with other options. Moreover, tumor-specific antigens that create clinically significant immunity are rare. Thus, future peptide vaccines will need improved CTL epitopes to increase immunogenicity.

Unlike peptide vaccines, DNA-based vaccines are not bound by the limitations of $\mathrm{MHC}$ restriction. Also, DNA vaccines allow for large-scale production and high purity, making DNA a more attractive vaccine strategy than the protein counterpart. Shi et al. [37] tested their DNA vaccine containing the HPV-16 E7 gene in a murine model. Knowing that the $\mathrm{E} 7$ gene and its encoded protein has a transforming activity, they selected for a mutant that had its zinc finger motifs disrupted. This markedly decreased the transforming activity of the protein. The disruption of the zinc finger also resulted in destabilization of the encoded E7 protein. From these murine model vaccination experiments, the mutant E7 DNA vaccine had a highly immunogenic effect, with marked elevation in E7-specific CTL response. Also, the mice were $100 \%$ protected from the tumor challenge. These results suggest that HPV-DNA vaccination is a viable option in human vaccine development. Furthermore, the observation that unstable E7 proteins can enhance the stimulation of CTL through MHC I-dependent antigen presentation may be clinically significant.

In contrast to attenuated viral vaccines, which produce immunity through altered growth cycles (ie, lowgrade infection), DNA vaccines do not have the ability to propagate, limiting their effectiveness, compared with standard attenuated viral vaccines such as mumps and rubella. In an attempt to enhance the immunity of DNAbased vaccines, heat shock protein genes have been fused to HPV gene vaccines (Table 3 ). This concept was initiated in HIV vaccine studies to elicit enhanced humoral and cellular immune response [38]. Chen et al. [39•] linked the full-length HPV-16 E7 gene and Mycobacterium tuberculosis heat shock protein 70 (hsp 70) and administered the vaccine in a murine model. The fused DNA vaccine increased the E7-specific CD8+ cells by at least 30-fold, compared with the wild-type E7 gene. Liu et al. [40••] also vaccinated the murine model with fused DNA HPV16 E7 CTL epitope and hsp 70. They did not vaccinate with a naked DNA but rather used the adenovirus as a delivery viral vector. By using the adenoviral delivery system, they were able to prime both the cellular and humoral immune response. This method appears to have stimulated both the MHC class I and II processing pathways in antigen-presenting cells. The Mycobacterium bovis bacille Calmette-Guérin (BCG) heat shock protein 65 (hsp 65) has also been used as a fused protein [41]. Unlike other heat shock protein-augmented vaccines with DNA, this was a fused protein vaccine with HPV-16 E7 and Mycobacterium BCG HSP. In a murine model, an augmentation in CTL by the presence of HSP was noted.

In addition to HSP, other genes have been fused to HPV to increase immunogenicity. For example, construction of a chimeric gene by linking the endosomal/lysosomal targeting signal with the HPV gene has been shown to increase the potency of this DNA vaccine. Ji et al. [42•] fused endoplasmic reticulum translocation signal peptide (Sig) and lysosome-associated membrane protein 1 (LAMP-1) to HPV-16 E7 to construct a chimeric DNA vaccine. Their hypothesis was that endosomal/lysosomal compartment rerouting can augment MHC II pathway presentation. Their murine vaccine trial showed an increased number of E7specific CD4+ helper T cells and greater E7-specific CTL activity. This study showed that, as with HSP, fusing of the E7 to the endosomal/lysosomal targeting signal may profoundly enhance the potency of the DNA vaccine. 


\begin{tabular}{|c|c|}
\hline Approach & Function \\
\hline Heat shock protein (HSP) & $\begin{array}{l}\text { Enhances the humoral and cellular immune response by being fused with HPV genes; } \\
\text { functions as an adjuvant }\end{array}$ \\
\hline Endoplasmic/lysosomal targeting domains & $\begin{array}{l}\text { Reroutes HPV E7 to be processed through MHC II presenting pathway; enhances } \\
\text { generation of CD4+ helper T cells and CD8+ CTLs }\end{array}$ \\
\hline Dendritic cells (DCs) & DCs pulsed with HPV-E7 gene induce generation of E7-specific CTL response \\
\hline GM-CSF & $\begin{array}{l}\text { Appears to induce maturation of dendritic cells; when linked with HPV antigen, acts } \\
\text { as a strong immunostimulatory signal, thus enhancing immunity }\end{array}$ \\
\hline Fms-like tyrosine kinase 3-ligand & $\begin{array}{l}\text { Has growth-stimulatory effect on dendritic cells; when linked with HPV antigen, } \\
\text { strongly enhances potency of DNA vaccine }\end{array}$ \\
\hline
\end{tabular}

Another approach to increasing the potency of DNA vaccines has been through the use of dendritic cells (DC), which are highly efficient antigen-presenting cells that activate immune responses. Wang et al. [43••] transfected DCs with the E7 gene to construct a DC-E7 vaccine, which was then administered in a murine model. Their study showed high E7-specific antibody, CD4+ T-helper cells, and CD8+ T-cell precursors. The potency of the vaccine was highest in the intramuscular route of delivery. Similarly, de Bruijn $e$ al. [44•] pulsed the DCs with HPV-16 E7 proteins and were able to elicit E7-specific CTL response. The vaccinated mice were then challenged with tumor cells, and the mice showed enhanced tumor protection.

In HIV vaccine studies, it has been demonstrated that granulocyte-macrophage colony-stimulating factor (GMCSF) gene linked with an antigen gene can augment the potency of DNA vaccination against HIV [45]. This has also been shown in hepatitis [46]. It appears that GM-CSF linked with an antigen acts as a strong immunostimulatory signal, enabling the maturation of the DCs and thus stimulating immunity. Chang et al. [47•] linked GM-CSF with HPV-16 E7 in a C75/BL/6 [H-2(b)]-derived melanoma cell line. These vaccine cells were then administered in the murine model. Marked antitumor activity was detected in the vaccinated mice, and E7-specific CTL activity was readily demonstrated. Similar to GM-CSF, another important molecule that is capable of growth-stimulatory effect on the DCs is Flt3 (Fms-like tyrosine kinase-3)-ligand (FL). Hung et al. $[48 \bullet \bullet]$ constructed a fused gene with HPV E7 and the extracellular domain of FL. This fusion vaccine showed greatly enhanced potency through the MHC I pathway.

E6 and E7 are the primary nonstructural HPV proteins in use for therapeutic vaccine development. L1 and L2 are used for the development of prophylactic vaccines. Chimeric vaccines involving both the capsid protein and E6 and E7 have been explored in an effort to develop a vaccine with both prophylactic and therapeutic potential. Greenstone et al. [49•] constructed a chimeric VLP (CVLP) with HPV-16 L1 and either E7 or E2 fused to the L2 minor capsid protein. A vaccine trial in a murine model showed that the vaccinated mice were protected from the tumor challenge. This indicates that, in addition to being able to generate humoral response, a CVLP containing the HPV early genes can also elicit cell-mediated immune response. Jochmus et al. [50] report a similar experience with HPV-16 L1 fused with E7.

\section{Conclusions}

HPV vaccine development for cervical cancer prevention is an active area of research. From the capsid protein-targeted vaccines to dendritic cell-stimulated DNA vaccines, many different approaches are being explored. With the recent report of a successful double-blinded, randomized clinical trial, many more trials of differing vaccines are sure to follow. Currently, vaccine strategies are rapidly advancing. In animal and human trials, many of these vaccines appear to enhance both the humoral and the cellular immune response. However, whether the promising results derived from animal models and early phase I human vaccine trials can translate into successful cervical cancer vaccine development with clinical significance is not known.

\section{References and Recommended Reading}

Papers of particular interest, published recently, have been highlighted as:

- Of importance

-. Of major importance

1. Murakami M, Gurski KJ, Steller MA: Human papillomavirus vaccines for cervical cancer. J Immunother 1999, 22:212-218.

2. Parkin DM, Pisani P, Ferlay J: Estimates of worldwide incidence from 25 major cancers in 1990. Int J Cancer 1999, 80:827-841.

3. Pisani P, Parkin DM, Bray F, Ferlay J: Estimates of the worldwide mortality from 25 cancers in 1990. Int J Cancer 1999, 83:18-29.

4. Walboomers JM, Jacobs MV, Manos MM, et al.: Human papillomavirus is a necessary cause of invasive cervical cancer worldwide. J Pathol 1999, 189:12-19.

5. Stoler MH: Human papillomaviruses and cervical neoplasia: a model for carcinogenesis. Int J Gynecol Path 2000, 19:16-28. 
6. Dupuy C, Buzoni-Gatel D, Touze A, et al.: Nasal immunization of mice with human papillomavirus type 16 (HPV-16) virus-like particles or with the HPV-16 L1 gene elicits specific cytotoxic T lymphocytes in vaginal draining lymph nodes. J Virol 1999, 73:9063-9071.

Intranasally administered VLP vaccine induced both humoral and cellular immune response in murine vaginal epithelia.

7. Bosch FX, Manos MM, Munoz N, et al.: Prevalence of human papillomavirus in cervical cancer: a worldwide perspective. J Natl Cancer Inst 1995, 87:796-802.

8. Human papillomaviruses: a compilation and analysis of nucleic acid and amino acid sequences. In Human Papillomaviruses 1996 Compendium: Glossary and Landmarks. Los Alamos, NM: Los Alamos National Laboratory; 1996:v-vi.

9. Scheffner M, Werness BA, Huibregtse JM, et al.: The E6 oncoprotein encoded by human papillomavirus types 16 and 18 promotes the degradation of p53. Cell 1990, 63:1129-1136.

10. Nead MA, McCance DJ: Activities of the transforming proteins of human papillomaviruses. In Human Tumor Viruses. Edited by McCance DJ. Washington, DC: American Society for Microbiology Press; 1998:225-251.

11. Gissman L: Human papillomaviruses and genital cancer. Semin Cancer Biol 1992, 3:253.

12. Schiller JT, Hidesheim A: Developing HPV virus-like particle vaccines to prevent cervical cancer: a progress report. J Clin Virol 2000, 19:67-74.

13. Stern PL, Brown M, Stacey SN, et al.: Natural HPV immunity and vaccination strategies. J Clin Virol 2000, 19:57-66.

14. zur Hausen H: Yohei Ito memorial lecture: papillomaviruses in human cancers. Leukemia 1999, 13:1-5.

15. af-Geijersstam V, Eklund C, Wang Z, et al.: A survey of seroprevalence of human papillomavirus types 16, 18 and 33 among children. Int J Cancer 1999, 80:489-493.

16. Lutzner MA: Papillomavirus lesions in immunodepression and immunosuppression. Clin Dermatol 1985, 3:165-169.

17. Garzetti GG, Ciavattini A, Buttini L, et al.: Cervical dysplasia in HIV-seropositive women: role of human papillomavirus infection and immune status. Gynecol Obstet Invest 1994, 40:52-56.

18. Petry KU, Scheffel D, Bode U, et al:: Cellular immunodeficiency enhances the progression of human papillomavirus-associated cervical lesions. Int J Cancer 1994, 57:836-840.

19. Ozsaran AA, Ates T, Dikmen Y, et al.: Evaluation of the risk of cervical intraepithelial neoplasia and human papilloma virus infection in renal transplant patients receiving immunosuppressive therapy. Eur J Gynaecol Oncol 1999, 20:127-130.

20. Kirnbauer R, Booy F, Cheng N, et al.: Papillomavirus L1 major capsid protein self-assembles into virus-like particles that are highly immunogenic. Proc Natl Acad Sci U S A 1992, 89:12180-12184.

21. Rose RC, Reichman RC, Bonnez W: Human papillomavirus (HPV) type 11 recombinant virus-like particles induce the formation of neutralizing antibodies and detect HPV-specific antibodies in human sera. J Gen Virol 1994, 75:2075-2079.

22. Pilacinski WP, Glassman DL, Glassman KF, et al.: Immunization against bovine papillomavirus infection. In Papillomaviruses: Ciba Foundation Symposium. Chichester: Wiley; 1986:136-156.

23. Christensen ND, Kreider JW, Kan NC, DiAngelo SL: The open reading frame $L 2$ of cottontail rabbit papillomavirus contains antibody-inducing neutralizing epitopes. Virology 1991, 181:572-579.

24. Breitburd F, Kirnbauer R, Hubbert NL, et al:: Immunization with virus-like particles from cottontail rabbit papillomavirus (CRPV) can protect against experimental CRPV infection. J Virol 1995, 69:3959-3963.

25. Christensen ND, Reed CA, Cladel NM, et al.: Immunization with virus-like particles induces long-term protection of rabbits against challenge with cottontail rabbit papillomaviruses. J Virol 1996, 70:960-965.
26. Suzich JA, Ghim S, Palmer-Hill FJ, et al.: Systemic immunization with papillomavirus $\mathrm{L} 1$ protein completely prevents the development of viral mucosal papillomas. Proc Natl Acad Sci U S A 1995, 92:11553-11557.

27. Kirnbauer R, Chandrachud L, O'Neil B, et al.: Virus-like particles of bovine papillomavirus type 4 in prophylactic and therapeutic immunization. Virology 1996, 219:37-44.

28. Nardelli-Haefliger D, Roden RBS, Benyacoub J, et al.: Human papillomavirus type 16 virus-like particles expressed in attenuated Salmonella typhimurium elicit mucosal and systemic neutralizing antibodies in mice. Infect Immun 1997, 65:3328-3336.

29. Revaz V, Benyacoub J, Kast WM, et al.: Mucosal vaccination with a recombinant Salmonella typhimurium expressing human papillomavirus type 16 (HPV 16) L1 virus-like particles (VLPs) or HPV 16 VLPs purified from insect cells inhibits the growth of HPV 16-expressing tumor cells in mice. Virology 2001, 279:354-360.

30. Rudolf MP, Nieland JD, DaSilva DM, et al.: Induction of HPV 16 capsid protein-specific human $T$ cell responses by viruslike particles. Biol Chem 1999, 380:335-340.

$31 . \bullet$ Harro CD, Pang YYS, Roden RBS, et al.: Safety and immunogenicity trial in adult volunteers of a human papillomavirus 16 L1 virus-like particle vaccine. J Natl Cancer Inst 2001, 93:284-292.

Report on the first double-blinded, randomized phase I VLP prophylactic vaccine trial in humans. In vaccinated human subjects, HPV-16-specific serum antibody titer increased by 40 -fold compared with those who were naturally seroconverted.

32. Borysiewics LK, Fiander A, Nimako M, et al.: A recombinant vaccinia virus encoding human papillomavirus types 16 and 18, E6 and E7 proteins as immunotherapy for cervical cancer. Lancet 1996, 347:1523-1527.

33. Steller MA, Gurski KJ, Murakami M, et al.: Cell-mediated immunological responses in cervical and vaginal cancer patients immunized with a lipidated epitope of human papillomavirus type 16 E7. Clin Cancer Res 1998, 4:2103-2109.

34. van Driel WJ, Ressing ME, Kenter GG, et al.: Vaccination with HPV 16 peptides of patients with advanced cervical carcinoma: clinical evaluation of a phase I-II trial. Eur J Cancer 1999, 35:946-952.

35. Ressing ME, van Driel WJ, Brandt RM, et al.: Detection of T helper responses, but not of human papillomavirus-specific cytotoxic T lymphocyte responses, after peptide vaccination of patients with cervical carcinoma. J Immunother 2000, 23:255-266.

36. Muderspach L, Wilczynski S, Roman L, et al:: A phase I trial of a human papillomavirus (HPV) peptide vaccine for women with high-grade cervical and vulvar intraepithelial neoplasia who are HPV 16 positive. Clin Cancer Res 2000, 6:3406-3416.

37. Shi $\mathrm{W}, \mathrm{Bu} \mathrm{P}$, Liu J, et al.: Human papillomavirus type $16 \mathrm{E} 7$ DNA vaccine: mutation in the open reading frame of $\mathrm{E} 7$ enhances specific cytotoxic T-lymphocyte induction and antitumor activity. J Virol 1999, 73:7877-7881.

38. Suzue K, Young RA: Adjuvant-free hsp70 fusion protein system elicits humoral and cellular immune responses to HIV-1 p24. J Immunol 1996, 156:873-879.

39. Chen CH, Wang TL, Hung CF, et al.: Enhancement of DNA vaccine potency by linkage of antigen gene to an HSP70 gene. Cancer Res 2000, 60:1035-1042.

A report on the fusion of HPV E7 and hsp 70 enhanced DNA vaccine potency.

$40 . \bullet$ Liu DW, Tsao YP, Kung JT, et al.: Recombinant adenoassociated virus expressing human papillomavirus type 16 E7 peptide DNA fused with heat shock protein DNA as a potential vaccine for cervical cancer. J Virol 2000, 74:2888-2894.

Use of a viral vaccine vector for fused HPV E7 and hsp 70 primed both the cellular and humoral immune response. 
41. Chu NR, Wu HB, Wu T, et al.: Immunotherapy of a human papillomavirus (HPV) type 16 E7-expressing tumour by administration of fusion protein comprising Mycobacterium bovis bacille Calmette-Guerin (BCG) hsp65 and HPV 16 E7. Clin Exp Immunol 2000, 121:216-225.

42. Ji H, Wang TL, Chen $\mathrm{CH}$, et al:: Targeting human papillomavirus type $16 \mathrm{E} 7$ to the endosomal/lysosomal compartment enhances the antitumor immunity of DNA vaccines against murine human papillomavirus type $16 \mathrm{E} 7$-expressing tumors. Hum Gene Ther 1999, 10:2727-2740.

Rerouting the HPV tumor antigen into the endosomal/lysosomal compartment enhanced the potency of this DNA vaccine.

43.• Wang TL, Ling M, Shih IM, et al.: Intramuscular administration of E7-transfected dendritic cells generates the most potent E7-specific anti-tumor immunity. Gene Ther 2000, 7:726-733.

E7-transfected dendritic cells were effective as vaccines in eliciting both humoral and cellular immune response in a murine model.

44. de Bruijn MLH, Schuurhuis DH, Vierboom MPM, et al.: Immunization with human papillomavirus type 16 (HPV 16) oncoprotein-loaded dendritic cells as well as protein in adjuvant induces MHC class I-restricted protection to HPV 16-induced tumor cells. Cancer Res 1998, 58:724-731.

Dendritic cells pulsed with HPV-16 E7 were used as a vaccine in a murine model. The vaccinated mice showed enhanced tumor protection.

45. Lee AH, Suh YS, Sung YC: DNA inoculations with HIV-1 recombinant genomes that express cytokine genes enhance HIV-1 specific immune responses. Vaccine 1999, 17:473-479.
46. Lee SW, Cho JH, Sung YC: Optimal induction of hepatitis C virus envelope-specific immunity by bicistronic plasmid DNA inoculation with the granulocyte-macrophage colony-stimulating factor gene. J Virol 1998, 72:8430-8436.

47. Chang EY, Chen CH, Ji H, et al.: Antigen-specific cancer immunotherapy using a GM-CSF secreting allogeneic tumor cell-based vaccine. Int J Cancer 2000, 86:725-730.

A fused vaccine with HPV E7 and GM-CSF, which stimulates the maturation of dendritic cells, showed both increased cellular immune response and antitumor activity.

48.• Hung CF, Hsu KF, Cheng WF, et al.: Enhancement of DNA vaccine potency by linkage of antigen gene to a gene encoding the extracellular domain of Fms-like tyrosine kinase 3-ligand. Cancer Res 2001, 61:1080-1088.

Linkage of HPV E7 with the extracellular domain of FL, which is capable of a growth-stimulatory effect on the dendritic cells, showed enhanced DNA vaccine potency.

49. Greenstone HL, Nieland JD, de Visser KE, et al.: Chimeric papillomavirus virus-like particles elicit antitumor immunity against the E7 oncoprotein in an HPV 16 tumor model. Proc Natl Acad Sci U S A 1998, 95:1800-1805.

Chimeric VLP with HPV early protein fused to the L2 capsid protein appears to elicit both humoral and cellular immune response.

50. Jochmus I, Schafer K, Faath S, et al.: Chimeric virus-like particles of the human papillomavirus type 16 (HPV 16) as a prophylactic and therapeutic vaccine. Arch Med Res 1999, 30:269-274. 